\title{
Germanica
}

$11 \mid 1992$

Le Conte dans les littératures germaniques du XXe siècle

\section{Elsa Beskow : le conte sur mesure}

Elsa Beskow: Märchen nach Mass

\section{Elena Balzamo}

\section{(2) OpenEdition}

Journals

Édition électronique

URL : http://journals.openedition.org/germanica/1343

DOI : 10.4000/germanica. 1343

ISSN : 2107-0784

Éditeur

Université de Lille

\section{Édition imprimée}

Date de publication : 1 janvier 1992

Pagination : 113-123

ISSN : 0984-2632

Référence électronique

Elena Balzamo, «Elsa Beskow : le conte sur mesure », Germanica [En ligne], 11 | 1992, mis en ligne le 07 février 2014, consulté le 06 octobre 2020. URL : http://journals.openedition.org/germanica/1343 DOI : https://doi.org/10.4000/germanica.1343

Ce document a été généré automatiquement le 6 octobre 2020.

(c) Tous droits réservés 


\title{
Elsa Beskow : le conte sur mesure
}

\author{
Elsa Beskow: Märchen nach Mass
}

\section{Elena Balzamo}

$1 \mathrm{Fr}$

2 Vers la fin du XIX ${ }^{e}$ siècle, le conte populaire existait en Suède sous deux " formes " principales, sans compter la tradition orale encore bien vivante dans la campagne suédoise. Les recherches des folkloristes débouchaient sur la publication croissante de recueils de contes transcrits avec la plus grande exactitude possible, souvent même en conservant les particularités dialectales. Précieux du point de vue scientifique, ils n'atteignaient presque jamais le grand public: leur valeur littéraire se trouvait compromise par le souci trop poussé de l'« exactitude ».

3 Cependant, jamais le genre du conte ne fut si en honneur qu'à cette époque. Mais le conte dont on se délectait avait peu à voir avec le vrai conte populaire. Le genre à la mode regroupait les récits dont les auteurs réarrangeaient les motifs du conte traditionnel (surtout du conte merveilleux et du conte facétieux), en sorte que tout en étant facilement identifiables comme dérivées du conte populaire, ces histoires-là, les contes littéraires de l'époque (konstsagor), avaient peu de choses en commun avec leur ancêtre populaire.

4 La prolifération de contes à la fin du XIX ${ }^{e}$ siècle coïncide avec l'expansion de la presse périodique pour enfants. Elle en est, en effet, la conséquence : le développement de l'instruction publique crée le besoin de donner aux enfants une lecture adaptée, de bonne qualité et - surtout! - bon marché. De nombreuses revues naissent en Suède pour répondre à cette demande. Les textes affluent de tous côtés. Beaucoup d'auteurs sont des instituteurs, ils réunissent souvent la compétence pédagogique et une solide connaissance de la tradition orale (la plupart d'entre eux enseignent dans les petites écoles de campagne). Quant à leur talent littéraire, il est variable...

5 La qualité des contes l'est aussi. Cela n'est guère étonnant : la majorité des auteurs sont des amateurs et les revues, afin de remplir les pages, acceptaient à peu près tout. Quelques grands noms surgissent de la masse: H. Nyblom, C. Granér, H. Kjellin, A. Smedberg, W. Stenström, A. Wallenberg, H. Österson. Célèbres au début du siècle, ils 
sombrèrent tous dans l'oubli après la première guerre mondiale. Depuis on les réédite parfois dans des anthologies, quelques uns de leurs contes font partie du répertoire classique de la littérature pour enfants, mais dans l'ensemble, ces auteurs sont bien oubliés et peu de Suédois connaissent aujourd'hui leurs noms.

6 À une exception: Elsa Beskow. Seule parmi tous ceux qui ont pratiqué ce genre, elle demeure vivante dans la mémoire collective. Elle est toujours rééditée et lue. Sa célébrité vient avant tout de ses livres illustrés pour les tout petits, mais aussi de ses contes proprement dits, où le dessin ne joue qu'un rôle secondaire. Inévitablement, on se trouve devant la question : comment expliquer cette particularité, cette célébrité durable au milieu de tant de gloires éphémères?

«... Après avoir réfléchi, je me suis dit qu'un vrai livre de contes devrait être précisément comme ce coffre. Il devrait y avoir des choses qui conviennent aux tout petits, et d'autres qui conviennent aux plus grands. Il devrait y avoir des choses utiles, des choses qui ne servent à rien, sauf à s'amuser, et aussi des choses bizarres dont on ne sait pas exactement que faire $»^{1}$. Cet extrait de la préface d'Elsa Beskow au Coffre $d u$ grenier du manoir (1926), résume assez bien son credo littéraire - credo qui aurait indigné ses prédécesseurs, illustres comme H.-C. Andersen ou Z. Topelius, et moins illustres comme la pléiade des auteurs du Sekelskiftet (fin XIXe début XXe siècles) pour qui la fonction éducative du conte ne faisait pas de doute. Le conte servait avant tout à faire passer un message pédagogique - et voilà un écrivain qui clame tout haut le contraire! Cette déclaration annonce une révolution - et pourtant, aucune révolution ne se produit. Les contes d'Elsa Beskow, inclus dans des anthologies ou lus dans les périodiques de l'époque, ne tranchent guère sur la masse des «konstsagor» (contes littéraires), leurs contemporains. Ses propres recueils, tout en laissant apercevoir un style très personnel, ne contiennent rien d'essentiellement différent des autres konstsagor. C'est qu'en fait, Elsa Beskow se trouve non pas au début d'un nouveau mouvement, mais achève un processus depuis longtemps amorcé.

En ce qui concerne sa doctrine pédagogique, la critique la considérait tour à tour comme réactionnaire ${ }^{2}$ et comme le modèle même du non-didactisme dans la littérature pour enfants ${ }^{3}$, selon qu'on la comparaît à Z. Topelius ou à A. Lindgren. Cependant, située dans son époque et jugée par rapport à elle, son œuvre montre aussitôt que ses idées pédagogiques sont aussi peu radicales que réactionnaires. Elle était parfaitement bien placée pour représenter le point d'équilibre dans la pédagogie de son temps. Liée aux milieux d'avant-garde par sa famille et son éducation (Ellen Key faisait partie de ce monde), elle épousa un pasteur, et fut ainsi introduite dans un environnement pratiquant et conservateur. Elle était aussi proche des problèmes d'éducation qu'Andersen leur était étranger, et aussi libre de tout engagement que Topelius était toujours engagé. Constamment en retrait, ne prenant jamais part à une querelle quelconque, elle s'est toujours gardée d'utiliser son art comme arme polémique - tout en y laissant transparaitre ses convictions. Son conte ne se pose ni comme un prolongement des manuels de classe, ni comme leur adversaire ou leur alternative. Il n'est pas pour autant «a-didactique » : pour Elsa Beskow - vu sa propre expérience l'éducation doit avoir lieu avant tout à l'intérieur de la famille et dans les rapports avec d'autres enfants. L'école est acceptée, un certain type d'école du moins, mais c'est elle 
qui est un complément de l'éducation donnée à la maison et non vice versa. Rien d'étonnant que les lignes de force de ses contes soient celles des relations familiales.

Tout compte fait, ce ne sont ni l'intensité de l'intérêt pédagogique d'Elsa Beskow, ni la force de son engagement qui lui permirent d'exprimer, sous la forme la plus pure, l'essence même du «konstsaga» de l'époque, mais précisément leur absence. Son conte est l'aboutissement du «konstsaga» suédois. Dans les années 1910, les idées esthétiques, qui se trouvaient à son origine et qui déterminaient sa forme et son contenu, étaient en train de perdre leur actualité, les problèmes n'étaient plus pertinents ou, en tout cas, ne faisaient pas partie des intérêts d'Elsa Beskow. Le conte littéraire se trouve chez elle débarrassé de son poids idéologique, allégé et comme désinvolte; son absence d'agressivité, sa gentillesse, sa tolérance témoignent moins d'une originalité de ses idées que d'un changement dans le statut du conte - désormais il ne s'agit que de le pratiquer en famille, pour l'agrément de ses proches.

Le processus qui s'achève avec Elsa Beskow, commence bien avant elle, au moment où le conte populaire «quitte » le folklore pour devenir un genre littéraire, c'est-à-dire à l'époque du Romantisme. À partir d'Andersen, la matière traditionnelle du conte est systématiquement corrodée : les sujets classiques sont modifiés, les motifs réarrangés, des accessoires - souvent comiques - ajoutés en quantité de plus en plus grande, l'histoire elle-même, d'habitude d'origine populaire, est systématiquement relativisée par la présence dans le texte du narrateur, qui - d'Andersen à Elsa Beskow, en passant par Topelius - affirme le droit de faire du conte ce que bon lui semble.

11 Le mélange de gravité moralisante et de gaieté nonchalante, souvent ironique, est le trait le plus marquant du conte littéraire tout au long du XIXe siècle. Le degré de désinvolture varie selon les auteurs: certains, comme H. Nyblom réussissent à préserver le sérieux, d'autres, comme Elsa Beskow ne cherchent pas à le faire, - on peut cependant affirmer que dans l'ensemble le conte littéraire n'a jamais été aussi peu sérieux qu'à l'époque (au début du siècle) où il était si à la mode. Comme si la désinvolture toujours croissante des contes devait contrebalancer la charge moralisatrice de plus en plus lourde. Pourtant les écrivains s'adonnent à ces amusements gratuits comme à contre-cœur, ne les admettant qu'au niveau du style, tandis que l'histoire elle-même reste porteuse d'un message éducatif parfaitement sérieux. Elsa Beskow fut celle qui comprit et accepta la gratuité du conte littéraire comme un de ses fondements, et qui l'utilisa comme un principe constructif dans plusieurs de ses histoires.

Pour tout lecteur attentif, les contes d'Elsa Beskow sont très facilement "identifiables». Les motifs, les situations peuvent sans difficulté être ramenés aux différentes sources populaires (ou littéraires). La raison de toute chose (1926) et Le garçon qui ne voulait pas aller à l'école appliquent le principe du conte cumulatif (AT 2025-2075); La peau de mouton de Klampe-Petter évoque les confrontations du "petit garçon » des Tales of the Stupid Ogre avec le "Grand Méchant » (ogre, troll, etc.); Runtbacka et Randå kra (1921) suivent pour une grande partie le schéma de la stupide querelle des époux 
(AT 1365) etc. Pourtant, son œuvre ne contient pas une seule adaptation de conte populaire en son entier.

13 Cela vient en partie du fait que parmi les grands auteurs de contes Elsa Beskow fut la première à ne pas avoir eu «l'expérience folklorique» que possédaient tous les écrivains du XIXe siècle. Sa famille était citadine, son milieu naturel, Stockholm, la seule tradition orale à sa disposition était celle de la ville. La connaissance des contes lui venait des livres. C'est pourquoi on trouve chez elle d'un côté une très forte imprégnation des modèles du conte populaire, des schémas extrêmement nets, et de l'autre côté, une prédilection visible pour les contes " déjà écrits ", qui constituent sa toile-de-fond, son "folklore à elle ", et qui lui sert de source d'inspiration et de thèmes pour variations.

Il n'est pas étonnant qu'elle ne fasse pas de différence entre les adaptations de Berg et les contes originaux d'Andersen et de Topelius - les deux devaient lui apparaître comme une sorte de "matière première ». Il est cependant fort probable qu'il s'agit là moins d'une particularité biographique que d'un phénomène plus général: d'une apparition du conte littéraire "au carré ", variété générique qui se nourrirait des éléments du conte populaire déjà assimilés et réorganisés d'une façon nouvelle, et qui serait aussi légitime que la précédente. S'il en est ainsi, un écrivain aurait à tenir compte d'un double système de références - le conte populaire et le conte littéraire du $\mathrm{XIX}^{\mathrm{e}}$ siècle, les deux étant désormais classiques.

La comparaison entre les textes d'Elsa Beskow et leurs modèles (populaires et littéraires) révèle une constante: les conflits sur lesquels reposent les histoires racontées, sont systématiquement atténués, le plan allégorique, si important chez les auteurs comme H.-C. Andersen, disparaît. Pour s'en rendre compte, il suffit de comparer L'aiguille à repriser d'Andersen avec L'épouvantail (1930), de Beskow ou alors Le perce-neige du grand Danois avec le Petit rosier beskowien. Dans Le perce-neige, la vie du perce-neige est présentée comme un échec sur le plan individuel (cueilli par les enfants, mis dans une lettre, oublié, jeté par terre, fourré dans un livre...), mais une réussite au niveau supra-individuel : vécu ainsi, le destin de la fleur devient comparable à celui d'un "grand poète " - cette découverte faite, le perce-neige n'éprouve plus d'amertume à l'égard de sa vie et en devient fier. Le conte, lui, devient parabole. Chez Elsa Beskow, les ambitions sont plus modestes : la canne de bambou, elle aussi, essuie l'échec dans ses entreprises: "Mais la canne de bambou, solitaire, se trouvait toujours là, droite et fière. L'année suivante, elle eut un autre fiancé, alors elle fut, elle aussi, heureuse et contente - et le conte peut se terminer vraiment bien $»^{4}$. La différence d'opinions, pour ne pas dire la polémique, avec Andersen, est évidente. Elsa Beskow refuse de transposer les conflits sur le plan allégorique, diminuant volontairement le diapason des lectures possibles. Son conte se referme, pour ainsi dire, sur lui-même : il redevient un univers clos, saris ouverture ou sortie sur un autre monde. Nous assistons au passage de la parabole à l'anecdote. Quel en est le sens?

Pourquoi ce refus de la multiplicité des interprétations ? Il s'agit, à notre avis, moins du refus d'affronter les problèmes "sérieux» que d'une tentative de les surprendre provisoirement - dans le conte. Le côté anecdotique devient donc très important : il permet de souligner qu'une telle solution ne convient qu'à telle ou telle situation 
concrète, qu'il n'y a pas de généralisation possible. En effet, toutes les histoires que raconte Elsa Beskow sont exceptionnelles - par opposition au conte populaire et au conte littéraire du XIXe siècle qui présentent volontiers des situations typiques, ou du moins traductibles sur le plan général.

Dès lors, on peut comprendre d'où vient cette multitude d'accessoires folkloriques, ces rois, ces princesses, ces objets magiques, toutes ces bribes de contes populaires qui ne forment cependant jamais un conte « entier ». Ils sont là pour donner l'apparence du typique à l'occasionnel, pour permettre aux «histoires qui se terminent bien» de devenir des «contes ». Ce sont les costumes du théâtre que revêtent les petits enfants pour que leurs conflits et leurs problèmes aient plus d'importance à leurs propres yeux, ainsi qu'aux yeux du lecteur. Le conte d'Elsa Beskow est le conte de la chambre d'enfants (barnkammaren), souvent un produit de leurs jeux (cf. Monsieur Klumpedump de la Klumpedonie, 1915) et il n'est pas étonnant que ses personnages soient surtout des enfants. Même là où l'emploi demande un adulte - comme dans le conte traditionnel celui-ci est, chez Elsa Beskow, considérablement rajeuni. Les rapports avec les parents, et conséquemment, le rôle de ceux-ci acquièrent une importance nouvelle, d'où le couple fréquent : le roi et la petite princesse.

L'enfant, chez Elsa Beskow, est tout à fait débarrassé du poids métaphysique qui souvent l'écrase dans les contes de ses prédécesseurs. Son statut reste néanmoins extrêmement élevé: grâce aux habits empruntés au conte populaire, les enfants deviennent princes et princesses; même quand il s'agit de petits paysans, le costume revêtu est presqu'aussi prestigieux, puisque ce sont bien les « petits cadets » du conte populaire.

19 L'atténuation des conflits, le rapetissement de l'univers, l'absence de cette menace sourde qui pèse sur le monde enchanté même chez des auteurs comme H. Nyblom, se répercutent inévitablement sur l'intrigue même du conte. Car depuis les premiers contes littéraires pour enfants d'Andersen, il existait une sorte d'incompatibilité entre les personnages d'enfants "réalistes", introduits par les auteurs, et l'intrigue traditionnelle empruntée au conte populaire. Ces petits enfants «réalistes » restaient en marge de l'histoire ; leur rôle était celui de spectateurs plus ou moins passifs. Dans les contes d'Elsa Beskow les enfants sont enfin à la hauteur de leurs tâches et deviennent de vrais héros de contes. Plus exactement : ce sont les tâches qui sont enfin descendues au niveau des personnages.

20 Cela amène une autre substitution importante. Ayant choisi comme protagoniste un personnage relativement faible, Elsa Beskow est obligée de lui donner un adversaire à sa mesure, quelqu'un qu'il puisse vaincre. Elle le fait, et voilà que dans ses contes les trolls se laissent facilement berner ou acheter (cf. La Peau de mouton de Klampe-Petter, 1930), le géant est vaincu par des petites filles armées d'un parapluie et d'un miroir (Tripp, Trapp, Trull et le géant Dum-dum), le dragon n'est qu'un jouet (Monsieur Klumpedump de la Klumpedonie), etc. Cette substitution est lourde de conséquences : dans le conte populaire, les personnages "négatifs » incarnent le Mal. Si ces personnages deviennent des similis et des jouets, où passe le Mal et que faire de l'esthétique binaire du conte? Car, si le Mal disparait, le Bien, lui aussi, n'a plus de raison d'être ou, plus 
exactement, plus de moyen d'expression artistique. Effectivement, chez Elsa Beskow il n'y a pratiquement pas de "méchants ", son conte se passe du personnage négatif !

Par conséquent, il n'est plus question d'« adversaires ", mais plutôt de " partenaires »; quant au conflit, ce n'est plus une épreuve de forces (physiques, comme dans le conte populaire, ou morales, comme dans le «konstsaga» de la plupart de ses contemporains), il devient une confrontation des opinions ou des volontés et est souvent résolu d'une façon verbale, au cours d'une conversation (cf. Anna-Stina et Pellele-Menteur, 1919). Ainsi, tout en maintenant une tension dans ses récits, Elsa Beskow réussit à en évacuer le « mal ».

Ce conte-jouet, ces exploits taillés sur mesure, ces oripeaux du conte populaire ne risquent-ils pas de se compromettre comme une copie, un pastiche trop franchement gratuits? Ce danger existe, mais il ne se concrétise guère, et ceci pour deux raisons.

Le premier lest qui empêche ce conte, en apparence trop léger, de « décoller ", est le quotidien qui en fait partie. La réalité de tous les jours, dans ses détails les plus menus, entre dans le conte et le rend convaincant, car elle imprègne non seulement ses parties "réalistes ", mais son univers entier. Monsieur Klumpedump de la Klumpedonie illustre bien cette technique: les jouets que les enfants fabriquent au début du récit, apparaissent dans le rêve du héros, qui est sa partie merveilleuse. Toutes les précisions sur la fabrication des jouets, que fournit l'auteur, non seulement «étoffent » la partie réaliste, mais sont également fonctionnelles dans le rêve, car elles déterminent l'apparence et le comportement des jouets devenus personnages. L'intrigue assez banale du conte traditionnel (le héros qui doit sauver une princesse), devient intéressante aussi bien par la façon dont elle est exposée, que par la tournure qu'elle prend à la fin et la morale qui en découle : l'important n'est pas de sauver la princesse, mais de bien faire ses jouets...

La deuxième source de la véracité des contes d'Elsa Beskow est dans la façon d'y introduire la nature.

Il n'est pas aisé d'isoler les paysages dans les contes d'Elsa Beskow, car, dans l'esprit de tout lecteur, ils sont indissolublement liés à ses dessins. La nature est pourtant également présente dans les textes eux-mêmes et sa présentation obéit à des règles tout à fait particulières. À la différence d'Andersen qui se borne à quelques détails suggestifs, ou de Topelius pour qui la description de la nature Scandinave constitue souvent une fin en soi et qui la regarde au cours de ses promenades (dans les bois, à la mer, etc.), Elsa Beskow étudie la nature à la loupe. Le domaine qui entre ainsi dans son champ visuel, est relativement petit, mais on en aperçoit d'autant mieux le relief. De même, la vie qui anime cette nature, revêt un caractère particulier ${ }^{5}$. Ce n'est pas le regard (de l'Enfant ou du Poète) qui transforme le monde quotidien - et donc la nature en un monde enchanté ; chez Elsa Beskow, la transformation est préalable au récit, et le regard enchanteur de l'enfant constitue le seul regard possible. La transformation (l'enchantement) a déjà eu lieu; elle n'a nul besoin d'être justifiée, ni même mentionnée. Le petit coin de jardin qui sert de décor à tant de ses histoires, ne fait pas " partie » de la réalité quotidienne, il ne s'oppose pas à elle, comme dans les contes de ses prédécesseurs, il la remplace - provisoirement, par jeu. 
Cette nature n'est pas non plus représentée par des images anthropomorphes, elle est anthropomorphe. C'est pourquoi l'opposition classique dans ce type de récits « enfant/ nature » cède ici la place à une symbiose. En sorte que la nature acquiert une double fonction: c'est sur elle que repose en grande partie la véracité du récit, son " réalisme », et c'est également elle qui lui fournit une dimension enchantée.

27 «Elle travaille tranquillement dans les limites du possible, de l'imaginable. Elle n'amène pas le lecteur dans le monde enchanté de l'incroyable, situé à côté de la réalité ; au lieu de cela, elle s'approprie la réalité et y introduit le conte ${ }^{6}$. Cette phrase de Tove Jansson contient une indication importante sur la proximité étonnante du monde enchanté d'Elsa Beskow. Ce monde est tellement limpide qu'on a du mal à le distinguer de la réalité, on en aperçoit les moindres détails. Ses frontières sont à peine perceptibles : il y a peu de formules qui en marquent les limites, il n'y a presque pas de récits-cadres, ni de passages d'un monde à l'autre. L'intégrité de ces contes repose sur le goût parfait avec lequel Elsa Beskow dose l'enchantement en fonction des situations « réelles » qu'elle décrit. Le conflit trop violent ne vient jamais troubler la réalité la plus quotidienne, les personnages enfants ne se voient jamais assigner des tâches audessus de leurs moyens, la rationalisation du merveilleux ne tourne pas en parodie, et inversement, une histoire trop réaliste ne subit jamais le poids d'un merveilleux trop traditionnel.

Grâce à cela Elsa Beskow reste, à notre avis, un maître insurpassable du genre, même si sa gloire vient avant tout de ses livres d'images ${ }^{7}$. Mieux que personne elle témoigna de l'impasse où se trouva le genre, dont les structures se figeaient de plus en plus, à partir des années 10 de notre siècle. Bien qu'elle en exploitât merveilleusement toutes les potentialités, elle n'a pas su le renouveler et jusqu'au milieu des années 40 le genre du conte littéraire garda en Suède la forme exquise, mais peu féconde, que lui avait donnée Elsa Beskow.

\section{NOTES}

1. - Elsa Beskow, Kistan på herrgårdsvinden, 1926, p. 13-14.

2. - G. Johansson, Elsa Beskow och Astrid Lindgren - en jämförelse, 3-betygsuppsats, Uppsala univ. 1966.

3. - S. Hammar, Elsa Beskow - författarinna och manniska, 3-betygsuppsats, Stockholms univ. 1955.

4. - Elsa Beskow, op. cit., p. 51.

5. - M. Sjögren, Elsa Beskow och hennes värld, Stockholm, 1983, p. 52.

6. - T. Jansson, «Sagan inom verkligheten», in Bonniers litterära magasin, 1959, p. 419.

7. - S. Hammar, op. cit., p. 44. 


\section{RÉSUMÉS}

Le point du départ de l'article est l'apparent paradoxe entre le credo littéraire iconoclaste d'Eisa Beskow et la surprenante conformité de ses contes aux canons du genre, tel qu'il se présente au début du XXe siècle. Son conte est l'aboutissement d'un long processus au cours duquel le conte littéraire affranchi de la tradition orale, est à la fois "relativisé » - grâce au recours systématique aux divers procédés stylistiques - et chargé d'un message didactique de plus en plus important. Conscient de cette tension, l'auteur cherche à épurer le genre; à cette fin, les modèles du conte traditionnel sont modifiés, les conflits atténués ou gommés, les personnages systématiquement rajeunis, l'opposition entre les adversaires incarnant le Bien et le Mal, fondamentale pour le conte traditionnel, est remplacée par le dialogue entre des " partenaires ». À la suite de ces opérations, le conte d'Eisa Beskow atteint une sorte d'équilibre, extrêmement stable grâce au savant dosage d'éléments "merveilleux» et "réalistes ». Ce qui permit à l'écrivain de pratiquer ce genre avec bonheur pendant plus de trente ans, mais qui fut - également - l'une des causes de l'impasse où le conte littéraire suédois demeura jusqu'au milieu des années 50 .

Der Ausgangspunkt des Artikels ist das scheinbare Paradox zwischen dem ikonoklastischen literarischen Credo der Elsa Beskow und der erstaunlichen Übereinstimmung ihrer Märchen mit den Gesetzen der Gattung, wie sie sich am Anfang des 20 Jahrhunderts präsentiert. Ihr Märchen ist das Resultat eines langen Prozesses, im Laufe dessen das Kunstmärchen, von der mündlichen Uberlieferung befreit, zugleich «relativiert» wird - dies dank der systematischen Anwendung der veschiedenen Stilverfahren - und mit einer didaktischen Botschaft von zunehmender Bedeutung versechen. Dieser Spannung bewusst versucht die Autorin die Gattung zu läutern; darum werden die Vorbilder des herkömmlichen Märchens umgeformt, die Konflikte gemildert oder ganz verwischt, die Figuren systematisch verjüngert. Die Konfrontation zwischen den das Gute und das Böse verkörpernden Gegenspielern, für das traditionnelle Märchen grundlegend, wird durch den Dialog zwischen Partnern ersetzt. Infolge dieser Bearbeitungen erreicht das Märchen von Elsa Beskow eine Art stabiles Gleichgewicht, dank der wohl dosierten Menge an wunderbaren und realistischen Elementen. Dies erlaubte der Autorin, diese Gattung über 30 Jahre mit Erfolg zu pflegen, zugleich aber war es einer der Gründe, warum das schwedische Kunstmärchen sich bis Mitte der fünfziger Jahre in einer Sackgasse befand. 